ARTICLE

\title{
LEAFY COTYLEDON1 expression in the endosperm enables embryo maturation in Arabidopsis
}

\author{
Jingpu Song (10) 1,2,3凶 , Xin Xie (i) 1,3, Chen Chen (1) 1,3,4, Jie Shu1,3,4, Raj K. Thapa (1) 1,3, Vi Nguyen', \\ Shaomin Bian (10) ${ }^{1,5}$, Susanne E. Kohalmi (iD ${ }^{3}$, Frédéric Marsolais (1D ${ }^{1,3}$, Jitao Zou (D) ${ }^{2 凶}$ \& Yuhai Cui (i) ${ }^{1,3 凶}$
}

The endosperm provides nutrients and growth regulators to the embryo during seed development. LEAFY COTYLEDON1 (LEC1) has long been known to be essential for embryo maturation. LEC1 is expressed in both the embryo and the endosperm; however, the functional relevance of the endosperm-expressed LEC1 for seed development is unclear. Here, we provide genetic and transgenic evidence demonstrating that endosperm-expressed LEC1 is necessary and sufficient for embryo maturation. We show that endosperm-synthesized LEC1 is capable of orchestrating full seed maturation in the absence of embryo-expressed LEC1. Inversely, without $L E C 1$ expression in the endosperm, embryo development arrests even in the presence of functional $L E C 1$ alleles in the embryo. We further reveal that $L E C 1$ expression in the endosperm begins at the zygote stage and the LEC1 protein is then trafficked to the embryo to activate processes of seed maturation. Our findings thus establish a key role for endosperm in regulating embryo development.

\footnotetext{
${ }^{1}$ London Research and Development Centre, Agriculture and Agri-Food Canada, London, ON, Canada. ${ }^{2}$ Aquatic and Crop Resource Development Research Centre, National Research Council of Canada, Saskatoon, SK, Canada. ${ }^{3}$ Department of Biology, Western University, London, ON, Canada. ${ }^{4}$ Molecular Analysis and Genetic Improvement Center, South China Botanical Garden, Chinese Academy of Sciences, Guangzhou, China. ${ }^{5}$ College of Plant Science, Jilin University, Changchun, China. ${ }^{凶}$ email: jsong267@uwo.ca; jitao.zou@nrc-cnrc.gc.ca; yuhai.cui@canada.ca
} 
S eed development in angiosperm is a complex process that is initiated by the double fertilization of egg and central cells with two sperm cells that generate the diploid embryo and the triploid endosperm, respectively ${ }^{1}$. The endosperm plays an essential role in seed development by nourishing the embryo via transferring maternal nutrients and growth regulators ${ }^{2}$. The development of embryo and endosperm depends on both parental genomes and is influenced by the communication and coordination of their genetic programs ${ }^{3}$. Although progress has been made ${ }^{2,4}$, the molecular interactions between the endosperm and the embryo during seed development are generally not well understood.

Many transcription factors have been shown to regulate seed development; one of them is a nuclear factor Y (NF-Y) transcription factor LEAFY COTYLEDON1 (LEC1) that has been identified as a key regulator of seed development ${ }^{5}$. In the embryo, LEC1 regulates seed development programs via combinatorial interactions with other transcription factors including the AFL B3 domain proteins, $A B I 3, F U S 3$, and $L E C 2$, which are all master regulators of seed maturation ${ }^{5-10}$. Null mutations in $L E C 1$ cause defective seed phenotypes, including short embryo axis, lessdeveloped cotyledons with anthocyanin accumulation, and desiccation intolerance ${ }^{11,12}$. Nonetheless, the lec1 homozygous plants rescued from mutant embryos did not exhibit any morphological abnormalities; neither were developmental paces and flowering time (Supplementary Fig. 1). As previously reported ${ }^{5}$, although there were no obvious morphological difference between wild type and the lec1-1 embryos from the globular to linear stages, embryos with purple cotyledons were observed from the lec1-1 mutant at early maturation (Supplementary Fig. 2). LEC1 expression has been detected not only in the embryo but also in the endosperm in several plant species, including Arabidopsis thaliana ${ }^{13}$, Brassica napus ${ }^{14}$, and soybean ${ }^{10}$, leading to speculation about a role of LEC1 in endosperm development ${ }^{5}$. However, no obvious morphological defects in the endosperm have been observed in lec 1 mutant seeds ${ }^{15}$. We hypothesized that the endosperm-expressed LEC1 may act as a molecular signal in the early communication between endosperm and embryo, and subsequently exerts its key roles in activating and regulating various embryo developmental programs. In the following sessions, we present the results of our genetic and transgenic experiments designed to test this hypothesis.

\section{Results}

Expression of LEC1 in the endosperm is required for seed maturation. To investigate if the expression of LEC1 in the endosperm was necessary for seed maturation, we generated seeds with unfertilized endosperms of lec1 genotype and fertilized diploid embryos of $L E C 1$ genotype using a genomic imprinting bypassing strategy (Fig. 1a), through which small but fully developed seeds could be produced when fis-class mutant flowers were crossed with $c d k a ; 1$ mutant pollens ${ }^{16,17}$. We first generated two double mutant lines, fis $2-6^{+/-} l e c 1-1^{-1-}$ and $c d k a ; 1^{+1-} l e c 1-1^{-/-}$. In the self-crossed siliques of the double mutants, half of the seeds were arrested at early development stages, as was seen in the self-crossed fis $2-6^{+/-}$and $c d k a ; 1^{+/-}$single mutants. The rest of the sibling seeds showed lec1 mutant phenotype as expected (Fig. $1 \mathrm{~b}$ and Supplementary Fig. 3a). To obtain seeds with lec1 endosperms and LEC1 embryos, we crossed fis2- $6^{+/}$lec1-1 $1^{-1-}$ flowers with $c d k a ; 1^{+l-}$ pollens (Fig. 1a). Meanwhile, crosses of fis $2-6^{+1-} \times c d k a ; 1^{+1-}$ and fis $2-6^{+1-} \times c d k a ; 1^{+/-} l e c 1-$ $1^{-/-}$were also conducted as positive controls, and that of fis2-6 $6^{+/-}$ lec $1-1^{-l-} \times c d k a ; 1^{+l-}$ lec1-1 $1^{-I-}$ as negative control (Supplementary Fig. $3 \mathrm{~b}-\mathrm{d}$ ). The $\mathrm{F} 1$ siliques had three types of seeds: normal size $\left(C D K A ; 1^{+/+}\right)$, small $\left(c d k a ; 1^{+/-}\right)$, and aborted (Fig. 1b-m). The normal sized seeds and small seeds were classified as not aborted
(NA) (Fig. 1c) and they were distinguished by the size of seed area (normal seeds: above $0.15 \mathrm{~mm}^{2}$, small seeds: $<0.1 \mathrm{~mm}^{2}$ ) as reported previously $^{16}$. Of note, genotype CDKA; $1^{+/+}$represents the normal sized seeds (Fig. 1d-g) which were wild type for both the FIS2 and CDKA;1 genes (maternal FIS2- $6^{+}$CDKA; $1^{+}$; paternal FIS2- $6^{+}$ $\left.C D K A ; 1^{+}\right)$since the maternal FIS2-6 ${ }^{+}$CDKA $; 1^{+}$paternal FIS2- $6^{+}$ $c d k a ; 1^{-}$genotype seeds aborted at the pre-globular stage (Fig. $1 \mathrm{~m}$ ). Genotype $c d k a ; 1^{+/-}$represents the small seeds (Fig. 1h-k) which were formed from maternal fis $2-6^{-}$CDKA; $1^{+}$and paternal FIS2- $6^{+}$ cdka; $1^{-}$since the maternal fis2- $6^{-}$CDKA; $1^{+}$paternal FIS2-6 ${ }^{+}$ $C D K A ; 1^{+}$genotype seeds aborted at the heart stage (Fig. 11). Further, the genotypes of the CDKA; $1^{+/+}$and $c d k a ; 1^{+/-}$seeds were confirmed by PCR-based genotyping at the CDKA; $1^{+} / c d k a ; 1^{-}$and the $L E C 1^{+} /$ lec $1^{-}$loci in the seedlings derived from each type of seeds (Supplementary Fig. 3e). Notably, the small seeds from the positive controls contained fully developed embryos (Fig. 1h, i). The small seeds from the crosses of fis $2-6^{+/-}$lec $1-1^{-1-} \times c d k a ; 1^{+l-}$, with $l e c 1^{-1-}$ endosperm and $L E C 1^{+/-}$embryo, on the other hand, showed defective embryo phenotype (Fig. $1 \mathrm{j}$ ) that was also observed in the small seeds from the negative control, with $l e c 1^{-1-}$ endosperm and $l e c 1^{-1-}$ embryo (Fig. 1k). Together, these results indicated that the expression of LEC1 in the endosperm was necessary for embryo maturation.

Haploid seeds with LEC1 endosperms and lec1 embryos develop normally. To test whether the expression of LEC1 in the embryo was required for seed maturation, we generated seeds with haploid lec1 embryos and normal LEC1 endosperms by pollinating flowers of a haploid induction line (SeedGFP-HI), which was generated by introducing a transgene expressing an altered form of CENH3 fused with GFP and also a transgene expressing GFP driven by the seed storage protein gene At $2 S 3$ promoter in the cenh3-1 line ${ }^{18,19}$, with lec1-1 pollen grains (Fig. 2a). We also conducted crosses of SeedGFP-HI flowers with wild type (WT) pollens to serve as control. From the SeedGFP$\mathrm{HI} \times$ lec $1-1$ and SeedGFP-HI $\times \mathrm{WT}$ crosses, we harvested the mature siliques containing a mixture of haploids and hybrid diploids in addition to a high frequency of aborted seeds (Fig. 2b). The diploid and haploid seeds were further hand-sorted based on their fluorescence patterns, as described previously ${ }^{18}$. The diploids with both embryo and endosperm inheriting the At2S3: GFP transgene in their maternal genome from the maternal parent SeedGFP-HI developed into seeds with uniform GFP signal, while the haploids with only the endosperm inheriting the maternal genome developed into seeds with mottled GFP fluorescence (Fig. 2c). From the crosses of SeedGFP-HI $\times$ lec1-1, haploid seeds with no lec1-1 seed defect (i.e., dark purple color) were obtained, which phenotypically resembled the haploid seeds from the SeedGFP-HI $\times$ WT crosses (Fig. 2c). To examine the embryo phenotypes, we dissected the diploid and haploid seeds. We observed that both diploid and haploid seeds collected from the SeedGFP-HI $\times$ lec1-1 and the SeedGFP-HI $\times$ WT crosses contained fully developed embryos. The phenotypes of those diploid and haploid embryos highly resembled WT embryos and were clearly distinguishable from the lec1-1 embryos (Fig. 2d). Diploid embryos were confirmed with the presence of GFP fluorescence and haploid embryos were GFP-negative (Fig. 2d).

The dissected diploids and haploids embryos were placed on MS agar plates to develop for subsequent genotype analysis. The diploid embryos were identifiable based on GFP signals observed at the centromeres in the root tip cells (Fig. 2e), because they inherited the CENH3-GFP transgene from the HI line. The haploid embryos only inherited genome information from the paternal plant lec1-1, thus displaying no GFP signal (Fig. 2e). To further validate the identities of the diploids and haploids, we examined the morphology and genotype of the plants developed 
a

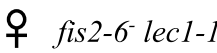

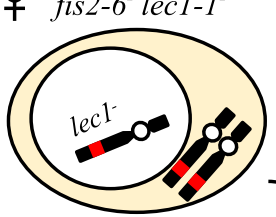

Egg cell fertilization

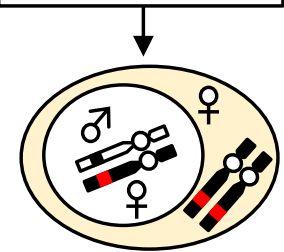

Em: $L E C 1^{+/} ;$Endo: lec $^{-/-}$ b

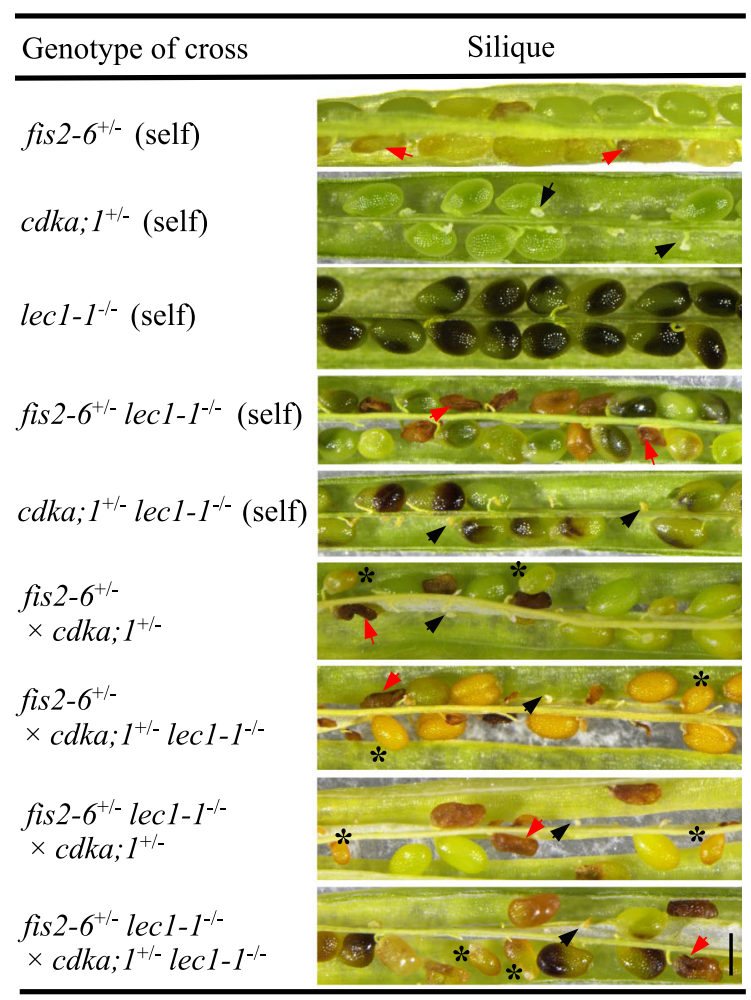

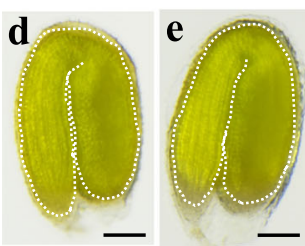

$\mathrm{LECl}^{+/ /}$
$\mathrm{LECl}^{+/-}$

$\mathrm{LECl}^{+/-}$

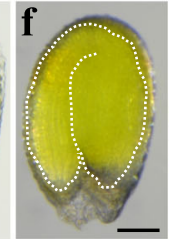

$$
\mathrm{LEC1}^{+/}
$$

$C D K A ; 1^{+1+}$

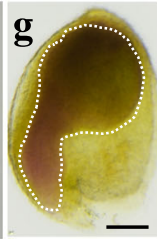

lec $1^{-/-}$

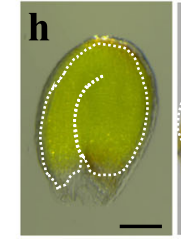
Endo: $L E C 1^{+/+}$
Em: $L E C 1^{+/+}$

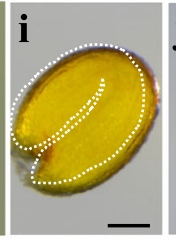

Endo: $\mathrm{LECl}^{+}$ Em: $L E C 1^{+/-}$

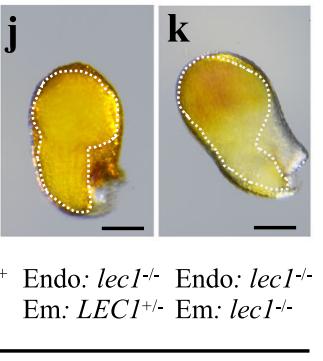

$c d k a ; 1^{+/}$
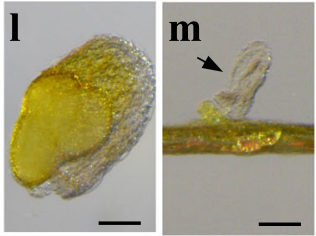

fis 2

cdka; $1^{-}$

Fig. 1 Loss-of-function of LEC1 in the endosperm disrupts seed development. a Cartoon showing a fis2-6- lec $1^{-}$female gamete crossing with a cdka; $1^{-} \angle E C 1^{+}$

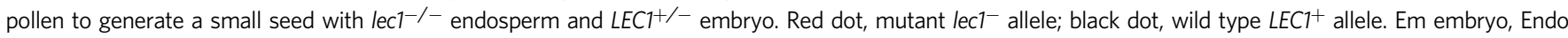
endosperm. b Green mature siliques collected from different genetic crosses. lec1 seeds display purple color. Arrows represent aborted seeds (red, fis ${ }^{-}$ phenotype; black, cdka; $1^{-}$phenotype); asterisks indicate small seeds generated from the fis $2-6^{-} \times c d k a ; 1^{-}$bypassing cross. Five siliques from each of the five crosses were examined. Scale bar: $500 \mu \mathrm{m}$. c Percentage of various progeny produced from the bypassing genetic crosses. CDKA; $1+/+$ indicate the normal sized wild type seeds, while $c d k a ; 1^{+/}-$indicate the small seeds. NA not aborted seeds, A aborted seeds, $\mathrm{n}$ number of seeds scored. $\mathbf{d}-\mathbf{k}$ Images of the CDKA; $1^{+/+}$ (normal size) and the $c d k a ; 7^{+/-}$(small) seeds with different genotypes produced from the bypassing genetic crosses. Five seeds from each genotype were examined. The crosses of fis $2-6^{+/-} \times c d k a ; 7^{+/-}$produce $\mathbf{d}$ type normal seeds and $\mathbf{h}$ type small seeds. The crosses of fis $2-6^{+/-} \times c d k a ; 7^{+/-}$lec $1-1^{-/-}$produce e type normal seeds and $\mathbf{i}$ type small seeds. The crosses of fis $2-6^{+/-}$lec1- $1^{-/-} \times \mathrm{cdka} ; 1^{+/-}$produce $\mathbf{f}$ type normal seeds and $\mathbf{j}$ type small seeds, while the crosses of fis2-6+/- lec1-1-/- $\times$ cdka; $7^{+/-}$lec1-1-/- produce $\mathbf{g}$ type normal size lec1 seeds and $\mathbf{k}$ type small seeds. I An example of aborted seeds produced by fis2- $6^{-}$. Five seeds were examined. $\mathbf{m}$ An example of aborted seeds produced by $c d k a ; 1^{-}$. Five seeds were examined. Scale bar: $100 \mu \mathrm{m}$.

from the dissected embryos. In comparison with the diploid plants, the haploid plants showed reduced stature featuring narrower and smaller leaves (Supplementary Fig. 4a), as previously observed ${ }^{19}$. During the reproductive phase, the diploid plants derived from the SeedGFP-HI $\times$ lec $1-1$ crosses produced normal size siliques that were filled with seeds segregating for the lec1-1 and wild type phenotypes (Supplementary Fig. 4b). In contrast, the haploid plants from the same crosses produced only a few siliques with 1-2 seeds of the lec1-1 genotype. Similarly, haploid plants from the SeedGFP-HI $\times$ WT crosses produced siliques containing 1-2 seeds of the wild type genotype
(Supplementary Fig. 4b). To confirm that the haploids from the SeedGFP-HI $\times$ lec1-1 cross only inherited the genome from the lec1-1 paternal parent, we genotyped both the diploid and haploid plants derived from the cross at the LEC1 locus and detected both the wild type LEC1 allele and the lec1-1 T-DNA allele in the diploid plants but only the lec1-1 T-DNA allele in the haploid plants (Supplementary Fig. 4c). These results further confirmed the identity of the haploid and the diploid seeds generated from the SeedGFP-HI $\times$ lec1-1 crosses.

The facts that the presence of wild type LEC1 allele inherited from the maternal parent in the endosperm of haploids as 
$\mathbf{a}$

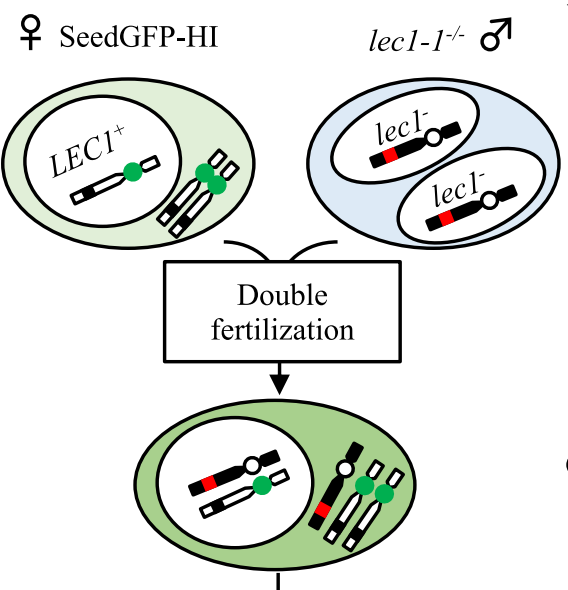

Em: Uniparental genome elimination
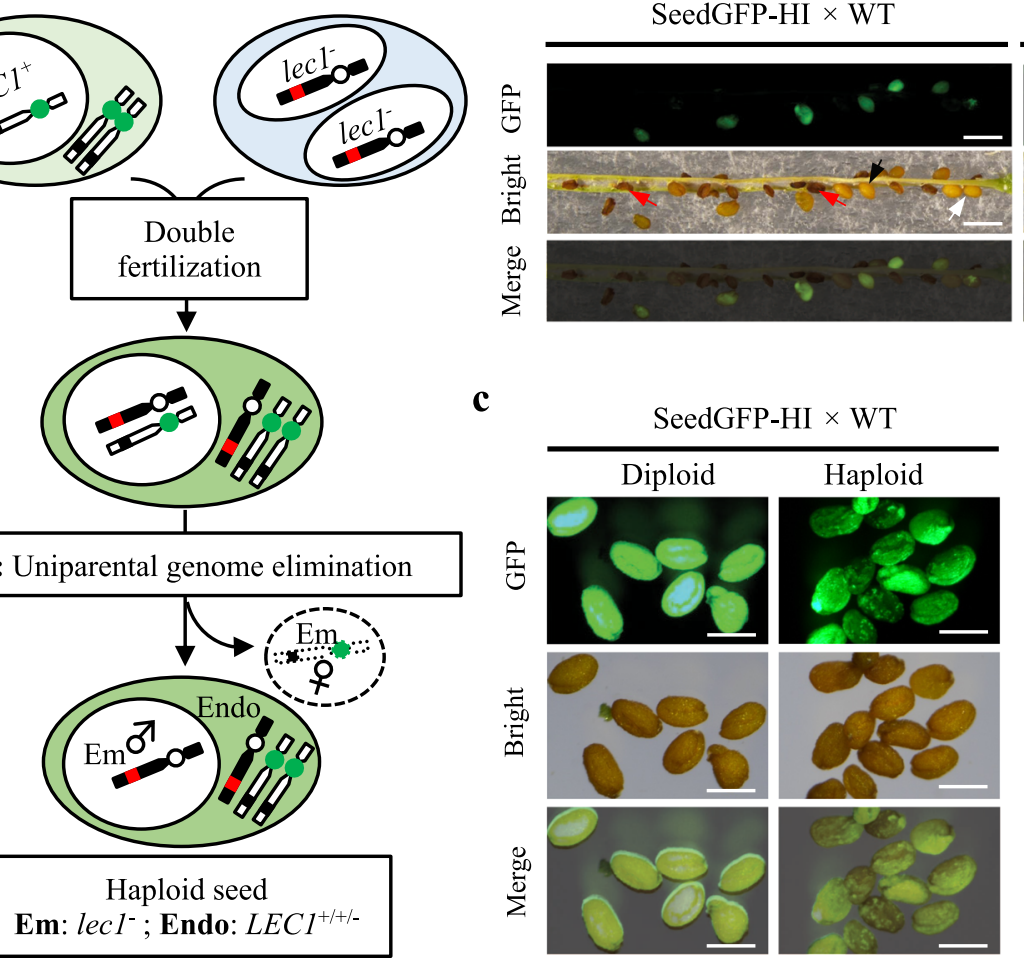

SeedGFP-HI $\times \operatorname{lec} 1-1^{-/-}$

c
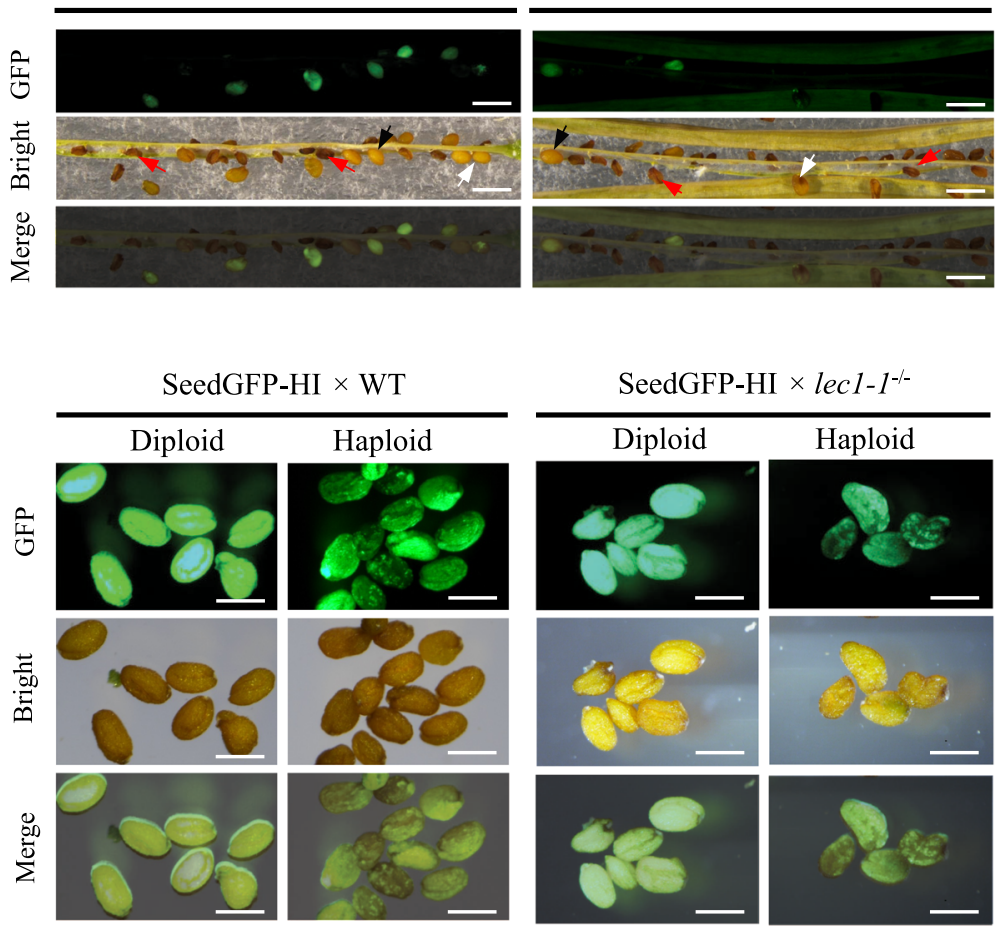

d

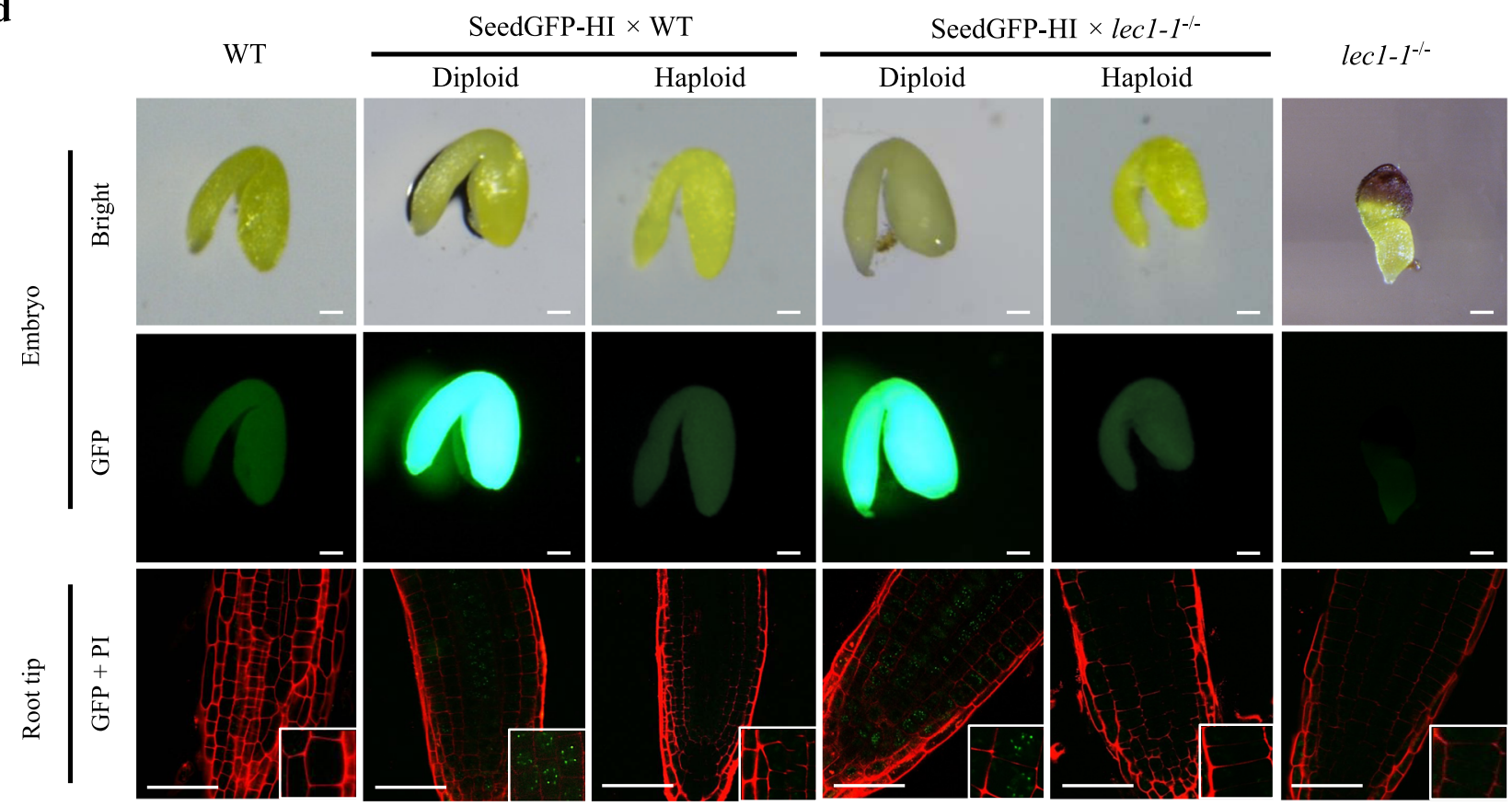

evidenced by the mottled seed GFP signals and that the haploid seeds did not exhibit any lec1-1 defective seed phenotypes such as purple cotyledons and short embryo axis (Fig. 2c, d) indicate that the seeds with LEC1 endosperm and lec1 embryo could develop normally through maturation. These results demonstrated that LEC1 gene expression in the embryo was dispensable for embryo maturation.

Expressing LEC1 exclusively in the endosperm rescues the lec1 seed phenotype. To further investigate whether an exclusive expression of $L E C 1$ in the endosperm can rescue lec1 seed defects, we employed two endosperm-specific promoters, proPHERES1 $(p P H E)^{20,21}$ and proZHOUPI ( $\left.p Z O U\right)^{22}$, to direct the expression of LEC1 in lec1 mutants. We built two endosperm-specific expression constructs, $p P H E:: L E C 1-G F P(\mathrm{PPL})$ and $p Z O U:: L E C 1-$ GFP (PZL), as well as controls that include $p L E C 1:: L E C 1-G F P$ (PLL), pPHE::ABI3-GFP (PPA), pZOU::ABI3-GFP (PZA), pPHE:: GFP (PPg), and $p Z O U:: G F P$ (PZg) (Supplementary Fig. 5). These constructs were introduced individually into embryo-rescued lec1-3 homozygous plants. From the T1 generation of PPL and 
Fig. 2 Fully developed haploid seeds with lec1 embryos and LEC1 endosperms. a Cartoon showing the genetic cross using the SeedGFP-HI line as female parent and lec1-1 as male parent to induce haploid progeny. Red dot: mutant lec1- allele; black dot: wild type $L E C 1^{+}$allele; green dot: weak centromeres CENH3-GFP from the HI parent; white dot: wild type centromeres. Em embryo, Endo endosperm. Note: the At2S3:GFP reporter in the SeedGFP-HI line is not shown in the cartoon. b Typical siliques containing GFP florescent seeds produced from the SeedGFP-HI $\times$ wild type $($ WT) or SeedGFP-HI $\times$ lec1-1 crosses. Approximately 30 siliques were produced from each type of the genetic crosses. Red arrows represent aborted seeds; black arrows indicate diploid seeds; white arrows indicate haploid seeds. Scale bar: $1000 \mu \mathrm{m}$. c Diploid and haploid seeds hand-sorted by their GFP signal patterns: seeds with uniform GFP signals (diploid) and seeds with mottled GFP fluorescence (haploid). At least four seeds from each genotype were examined for each replicate. This experiment was repeated three times independently. Scale bar: $500 \mu \mathrm{m}$. d Hand-dissected embryos from the mature diploid and haploid seeds produced from the crosses. Seed GFP florescence were detected under UV lights. Three embryos from each genotype were examined. Scale bar: $100 \mu \mathrm{m}$. e Detection of GFP signals in the centromere of root tip cells under confocal microscope. Insets: magnified areas of root cells indicated. Cell walls stained with propidium iodide (PI) are shown in red. Confocal images are shown as the merged channel of GFP and PI. Three embryos of each seed type were examined. This experiment was repeated three times independently. WT and lec1-1 embryos were used as control. Scale bar: $50 \mu \mathrm{m}$.

PZL plants, $67 \%$ and $74 \%$ of the seeds (T2), respectively, were rescued to normal phenotype (Fig. 3a). Among the lines introduced with the control constructs, $79 \%$ of the embryos from the PLL plants showed normal phenotype, but none were found in the PPA, PPg, and PZA lines (Fig. 3a). We then tested the germination of $\mathrm{T} 2$ seeds to test if the transgenic seeds were desiccation-tolerant. As shown in Fig. $3 \mathrm{~b}$ and Supplementary Fig. 6, seeds from the PPL, PZL, and PLL lines germinated successfully, while those from PPA, PPg, and PZA succumbed to desiccation. Morphologically, the mature seeds of PPL, PZL, and PLL were indiscernible from that of wild type, but the PPA, PPg, and PZA seeds resembled that of lec1-3 (Fig. 3c-m). The PPL, PZL, and PLL seeds possessed wild type levels of storage proteins (12S and 2S) while PPA, PPg, and PZA exhibited much lower levels similar to that of lec1-3 (Fig. 3n). In addition, the same transgene constructs were also introduced into the lec1-1 plants in parallel and analogous results were obtained (Supplementary Fig. 7). Hence, LEC1 directed to be expressed solely in the endosperm was fully capable of complementing lec1 mutant seed in embryo morphology, seed germination, and storage protein accumulation. These transgenic experiments, in combination with the above described genetic evidence obtained from the haploid seed experiment, established that exclusive expression of LEC1 in the endosperm is sufficient to capacitate normal embryo development.

The onset of LEC1 expression occurs first in the endosperm. To decipher the initiation and the mode of action of the endospermexpressed LEC1, we closely monitored GFP signals in the developing seeds of PLL, PPL, and PPg from the zygote stage onward, to maturation stage (Fig. $4 \mathrm{a}-\mathrm{u}$ ). Pollen grains and ovules in the PLL before fertilization were also examined, and no GFP signal could be detected (Supplementary Fig. 8a, b). GFP signals were first emerged from fertilized central cell (endosperm) nuclei in the PLL, PPL, and PPg seeds at the zygote stage (Fig. $4 \mathrm{a}-\mathrm{c}$ and Supplementary Fig. 8c-e). By the two-cell stage, GFP signals began to appear in the pro-embryo of PLL, in both the endosperm and the pro-embryo of PPL, but restricted to endosperm only in the PPg seeds (Fig. 4d-f and Supplementary Fig. 8f-h). In the PLL embryos, GFP signals were observed from the globular to bent stages, but not at the maturation stage (Fig. $4 \mathrm{~g}, \mathrm{j}, \mathrm{m}, \mathrm{p}, \mathrm{s}$ ). Similarly, the PPL embryos exhibited GFP signals at the globular, heart, linear, and bent stages, but not the maturation stage (Fig. $4 \mathrm{~h}, \mathrm{k}, \mathrm{n}, \mathrm{q}, \mathrm{t}$ ). Notably, strong signals were present in the suspensors of PLL and PPL embryos (Fig. 4d, e, g, h, j, k). Similar to observations from the PPL, GFP signals were also detected in developing embryos of the PZL seeds (Supplementary Fig. 8i, j). In contrast, there was no GFP signal in the PPg embryos at any stages (Fig. 4c, f, i, l, o, r, u). In the PPA seed, consistent with its lec1 mutant embryo phenotype, GFP signals were only detected in the endosperm nuclei at the pre-globular stage, but not in the embryos (Supplementary Fig. 9).

To determine whether the LEC1 in the embryos of PPL was mobilized from the endosperm, we further generated a PPL-GFP 3 line expressing LEC1 fused to 3 copies of GFP (Supplementary Fig. 10a). Such fusion proteins with multimeric GFPs have been employed successfully by others to restrict the mobility of mobile transcription factors ${ }^{23,24}$. LEC1-GFP ${ }_{3}$ signals were observed in the endosperm nuclei at the pre-globular stage, but not in the embryos at the pre-globular, globular, heart, or linear stages (Supplementary Fig. 10b). These results suggest that the LEC1 fusion protein was restricted to the endosperm in the PPL-GFP 3 seeds. Consistent with the lost mobility of LEC1-GFP 3 , the fusion gene failed to rescue the lec1-1 seed defects (Supplementary Fig. 10c).

In addition, we generated another control, the pAtML1::LEC1GFP lec1-1-/- (PML) line, employing the embryo-specific AtML1 promoter to drive the exclusive expression of LEC1 in the embryo $^{25}$. As expected, expression of the LEC1-GFP fusion in the PML was only observed in the embryos, and it could successfully rescue the lec1-1 seed defects (Supplementary Fig. 11). This result is consistent with earlier observations that the lec1 mutant endosperms do not show any obvious defect ${ }^{5}$, and is also in line with our observations presented above (Supplementary Fig. 2).

In light of the endosperm specificity of $p P H E$, these results signified that in the PPL seeds the endosperm-expressed LEC1GFP was trafficked to the embryo from its expression origin, the endosperm, to enable embryo maturation. Such an scenario is further supported by our data from the PPL-GFP 3 line. These results show that LEC1 was mobilized from the endosperm to the embryo at very early stages of seed development.

The LEC1 protein enters the embryo from the endosperm. To investigate whether LEC1 was mobilized from the endosperm to the embryo in the form of RNA or protein, we performed RTqPCR analyses to determine if there was any LEC1-GFP mRNA in the embryos. First, we measured the relative expression of GFP in the homozygous PLL, PPL, PPg, and WT (negative control) whole seeds at the linear stage, confirming the presence of GFP transcripts in the PLL, PPL, and PPg seeds (Fig. 4v). We then measured the GFP mRNA in the linear stage embryos of PLL, PPL, using PPg and WT as negative controls since they had no GFP signal in the embryos. GFP mRNA was detected in the PLL embryos but not in the PPL embryos (Fig. 4w). This result, when combined with the observation of clear presence of LEC1-GFP signals in the PPL embryos, strongly suggests that LEC1 was transported in the form of protein from the endosperm to the embryo. Such a notion is also consistent with our observation that there was no GFP signal in the PPL-GFP ${ }_{3}$ embryos.

We lastly examined the effectiveness of endosperm-synthesized LEC1 in activating embryo maturation genes known to be 
a

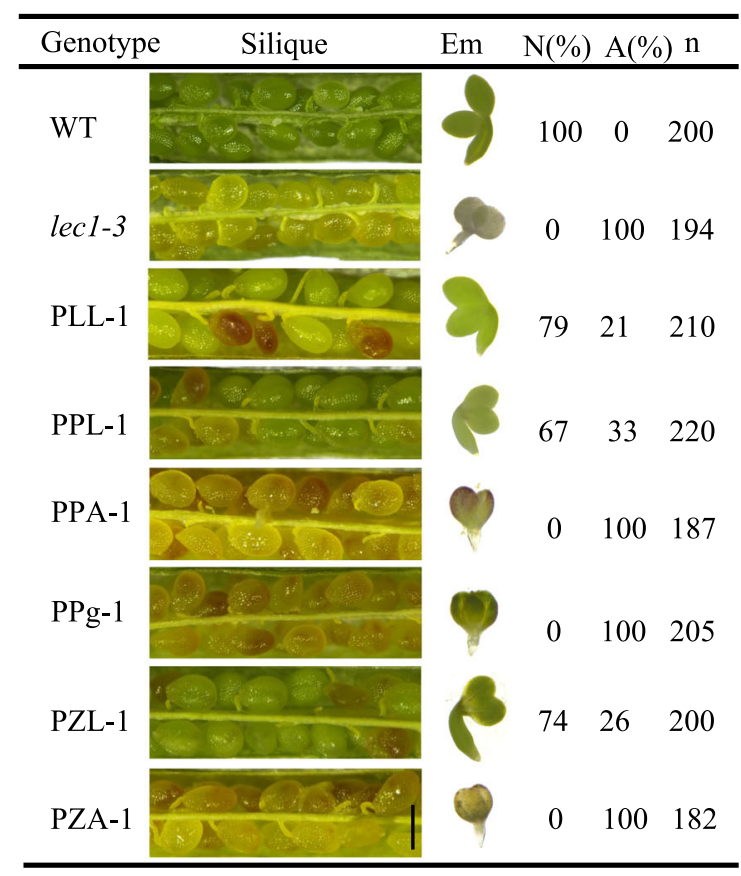

b

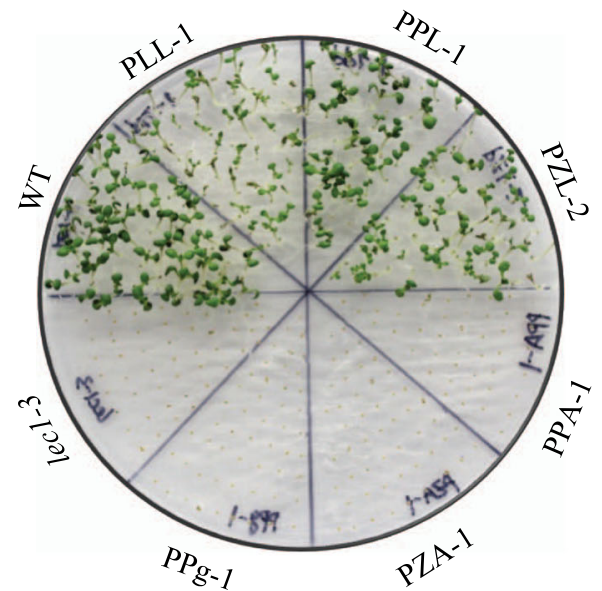

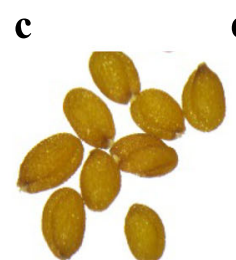

WT

g

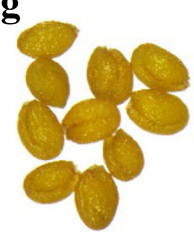

PPL-1 d

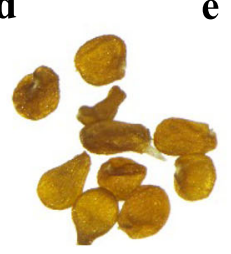

lec1-3

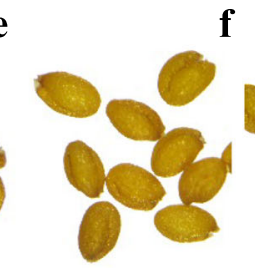

PLL-1

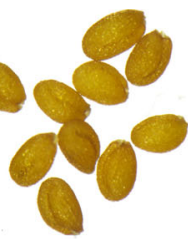

PLL-2

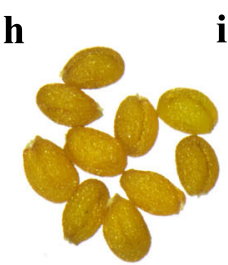

PPL-2

i

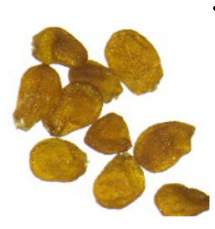

j

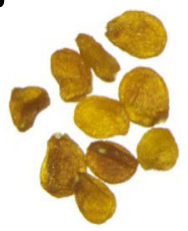

PPA-1

PPg-1

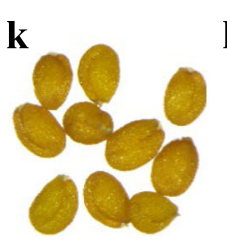

PZL-1 l $\mathbf{m}$

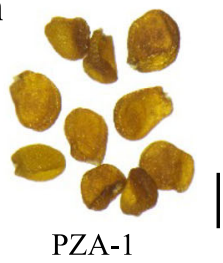

n

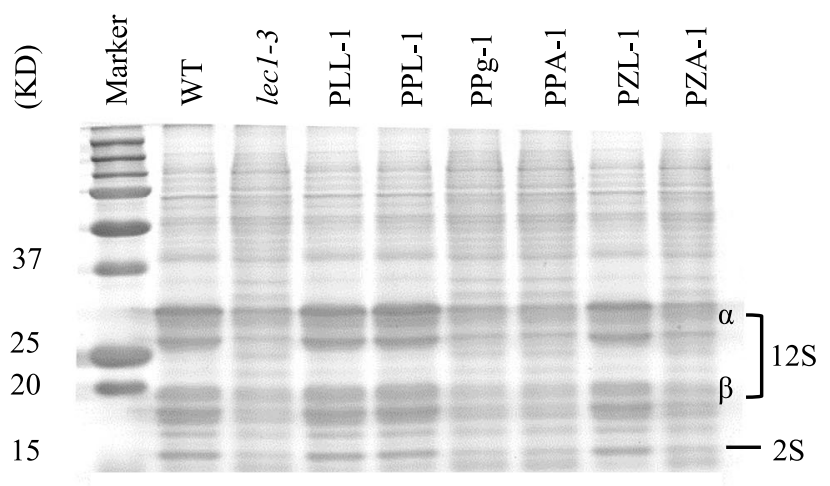

Fig. 3 Gain-of-function of LEC1 in the endosperm rescues lec1-3 mutant seed phenotype. a Green mature siliques collected from T1 plants with different transgenes in the lec1-3 background. Siliques of T1 plants PLL-1 (pLEC1::LEC1-GFP lec1-3-/-) , PPL-1 (pPHE:::LEC1-GFP lec1-3-/-), and PZL-1 (pZOU::LEC1-GFP lec1-3 ${ }^{-/-}$) contain two types of seeds: [WT] seeds and [lec1-3] seeds. PPA-1 (pPHE::ABI3-GFP lec1-3-/-), PPg-1 (pPHE::GFP lec1-3-/-), and PZA-1 (pZOU:: $A B I 3-G F P$ lec1-3-/-) only produce defective seeds (lec1-3). [WT] indicates WT phenotype, [lec1-3] indicates lec1-3 phenotype. At least five independent individual transgenic lines were examined for each construct transformation. Images of a typical embryo from each line are shown on the right next to the images of the siliques. WT (Ler-0) and lec1-3 are used as control. N normal seeds, A abnormal seeds (lec1-3), n number of seeds scored. Em embryo. Scale bar: $500 \mu \mathrm{m}$. b Germination of seeds with different transgenic backgrounds at day 7. Fifty seeds were used for one biological germination test. This experiment was repeated three times independently. c- $\mathbf{m}$ Phenotypes of mature seeds from each of the transgenic backgrounds as indicated. Ten seeds from each genotype were examined. Scale bar: $300 \mu \mathrm{m}$. $\mathbf{n}$ SDS-PAGE gel image showing the $2 S$ and $12 S$ storage proteins in seeds from each of the transgenic backgrounds. This experiment was repeated three times independently.

downstream target genes of $\mathrm{LEC1} 1^{5,26}$, including the AFL B3 transcription factor genes $A B I 3$, FUS3, and LEC2. Through analyzing a set of LEC1 ChIP-seq data from a previous study ${ }^{10}$, we were able to verify the LEC1 occupancy on the promoters of $L E C 2$, FUS3, ABI3 and LEC1 (Supplementary Fig. 12). We then measured the relative expression levels of $L E C 2, F U S 3$, and $A B I 3$ in the linear stage embryos of PLL, PPL, and PPg. As shown in Fig. $4 \mathrm{x}$, similar levels of $L E C 2, F U S 3$, and $A B I 3$ expression was detected in the PPL and PLL embryos, but almost negligible in the PPg embryos. These results demonstrated that the LEC1 protein synthesized in the endosperm adequately activates the seed development programs including the maturation genes without de novo LEC1 synthesis in the PPL embryo.

\section{Discussion}

Our study uncovers a mode of action of $L E C 1$ that is initially expressed in the endosperm but subsequently in the form of LEC1 protein mobilized to the embryo as a molecular signal (Fig. 5). Given the LEC1-GFP strong signals being detected in the suspensors, we conjecture that the route of trafficking is via the suspensor, which has long been suspected of mediating symplastic transfer of metabolites and proteins from the endosperm to embryo ${ }^{2}$. The endosperm-synthesized LEC1 protein, once transported into the embryo, can subsequently trigger the expression of $L E C 1$ in the embryo and ultimately the LEC1-regulatory network to enable normal seed development. Such an scenario is also consistent with the postulated activity of LEC1 as 


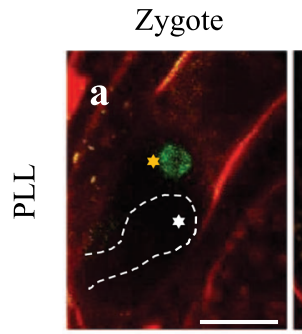

Two-cell

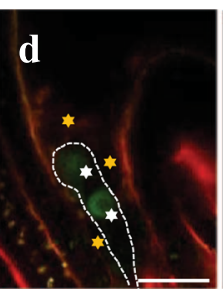

Globular
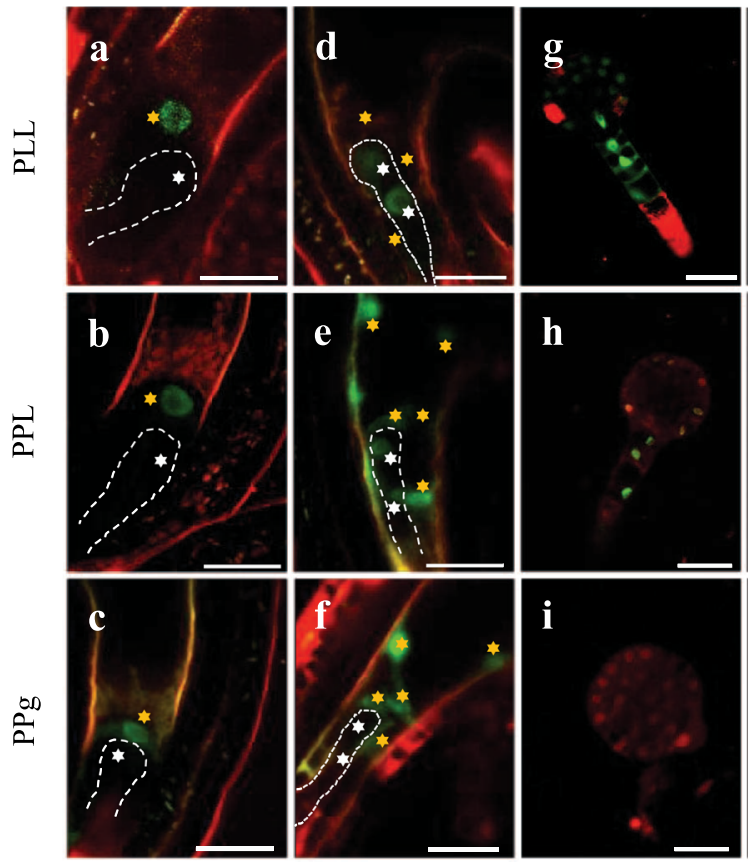

$\mathbf{W}$
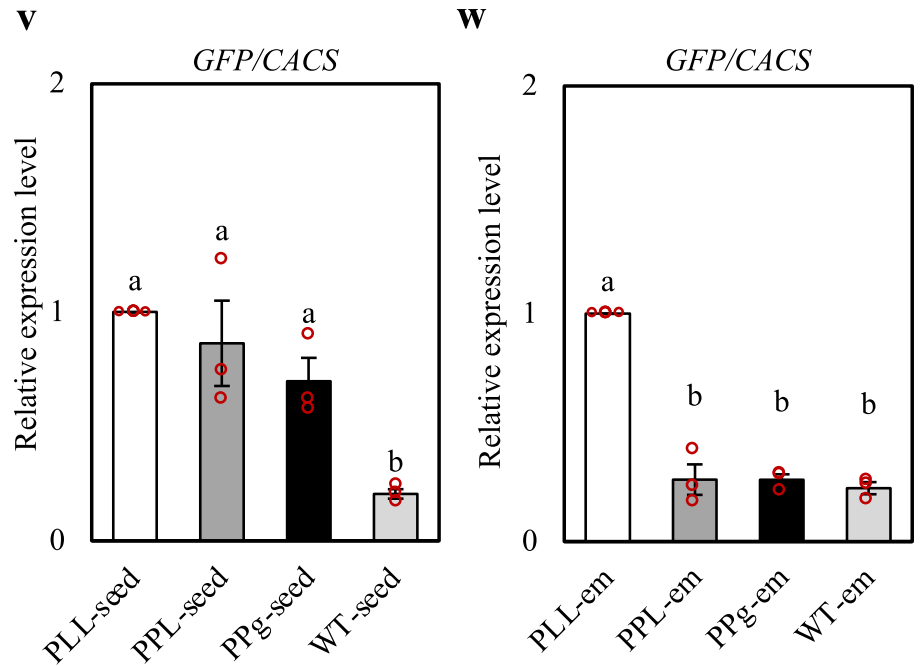

Heart
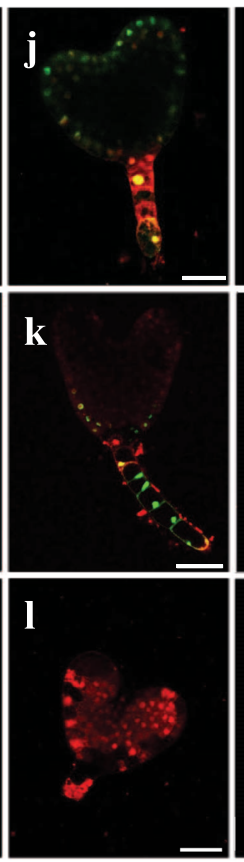

Bent
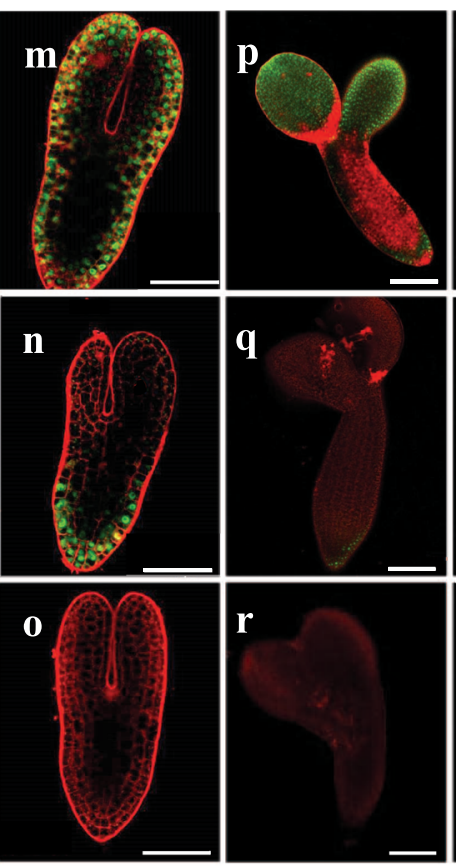

Maturation

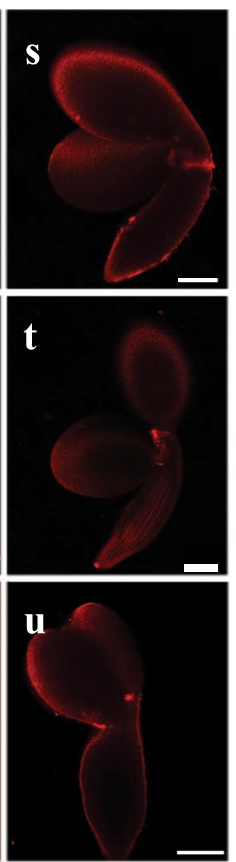

$\mathbf{X}$

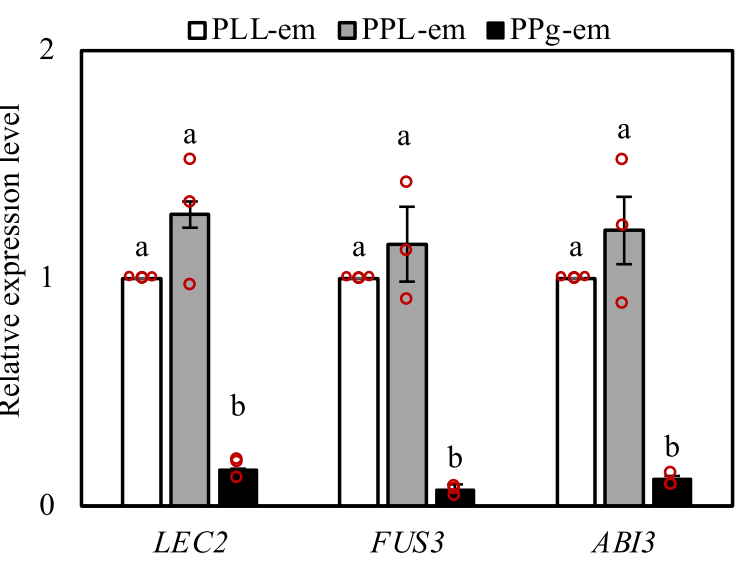

Fig. 4 Endosperm-expressed LEC1 enters embryo to regulate seed maturation genes. a-u Localization patterns of GFP signals in PLL, PPL, and PPg seeds at various seed development stages from zygote to maturation as indicated. Five seeds or embryos at each stage from each line were examined. Embryos (a-f) are outlined in white dash lines for clarity. Amber stars indicate endosperm nuclei and white stars indicate embryo nuclei. GFP signals are shown in green. Cell walls stained with PI are shown in red. Shown here are merged confocal images from GFP and PI channels. Scale bars: $20 \mu \mathrm{m}(\mathbf{a}-\mathbf{I}) ; 50 \mu \mathrm{m}(\mathbf{m}-$ o); $100 \mu \mathrm{m}(\mathbf{p}-\mathbf{u})$. v Relative expression of GFP in the whole seeds of PLL, PPL, PPg, and WT at linear stage. w Relative expression of GFP in the embryos (em) of PLL, PPL, PPg, and WT seeds at linear stage. $\mathbf{x}$ Relative expression of $L E C 2, F U S 3$ and ABI3 in the embryos (em) of PLL, PPL, PPg seeds at linear stage. $\mathbf{v}-\mathbf{x}$ a to $b$ indicate statistical difference with one-way ANOVA followed by the post-hoc Tukey multiple comparison tests ( $p<0.05)$. The CACS gene was used as an internal control. Values are mean \pm standard error of three biological replicates.

a pioneer transcription factor ${ }^{27}$ and the notion that the chromatin environment in the endosperm is distinct from that in the embryo ${ }^{3}$. Seed genes, particularly those pertinent to seed maturation, are believed to be repressed during vegetative growth by the polycomb proteins-mediated chromatin condensation ${ }^{28-30}$. How they get reset during seed development has been a puzzling question. Our findings provide a plausible explanation for the reprograming of these genes in the new generation (i.e., seed). It is conceivable that, due to the favorable chromatin and cellular environments in the endosperm, $L E C 1$ becomes activated there shortly after fertilization and enters the embryo, where it overcomes the repressive chromatin context to trigger the de-repression of seed genes including itself.
Why would a functional LEC1 copy remain expressed in the embryo, given that its expression in the endosperm seems to be enough to ensure proper seed development? It would be tempting to speculate that one advantage would be to have more-thanenough LEC1, as an enhanced mechanism, to enable/doublesecure the normal embryo development, a vital process for a plant's survival. Another puzzling question is why LEC1 expression in the embryo is normally dependent on LEC1 protein provided to the embryo by the endosperm. We would speculate that such an arrangement allows a spatial separation of the embryo and its key regulator, thus adding a new layer of control in the regulation of embryo development. This would make sure 


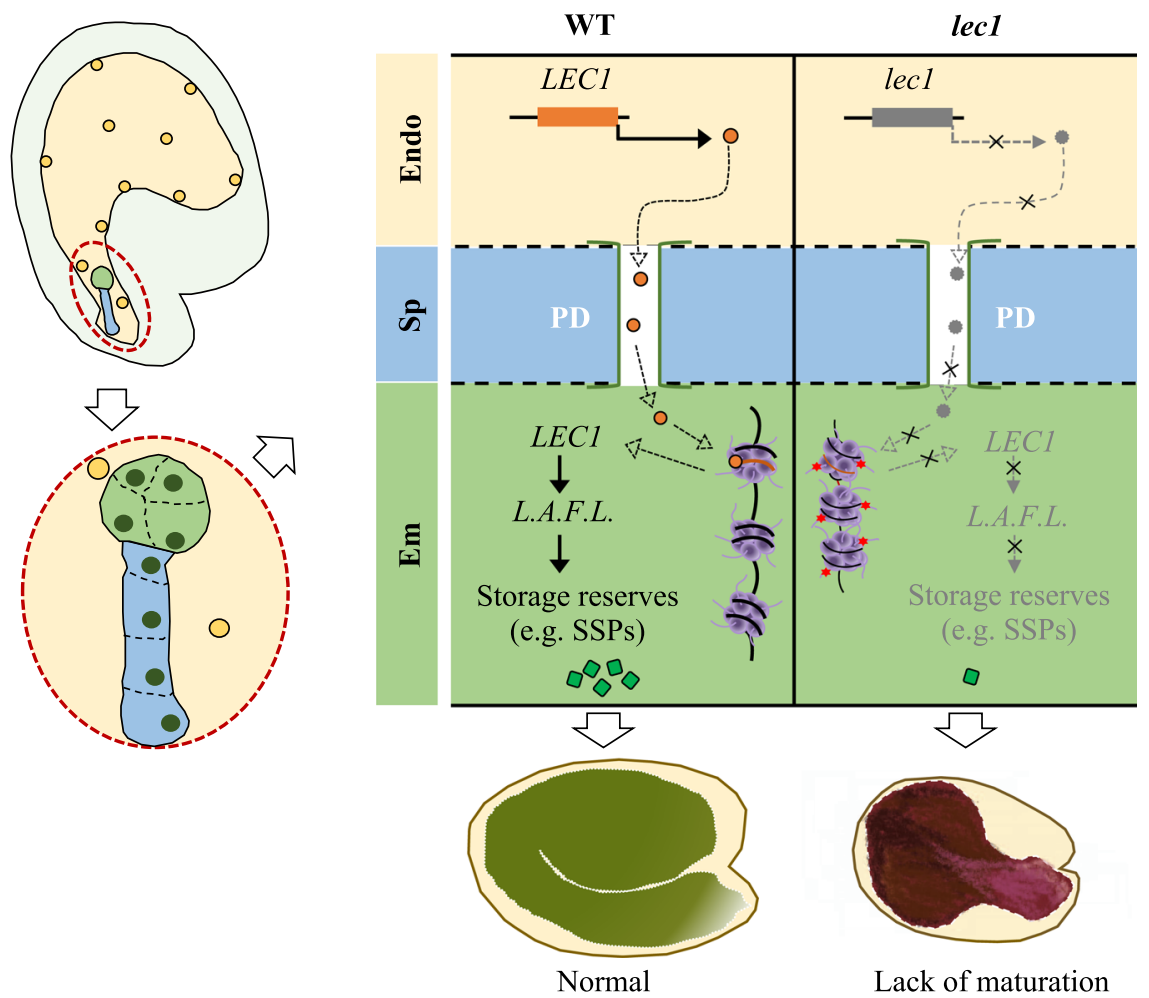

Fig. 5 A model depicting the regulation of seed maturation by the mobile transcription factor LEC1. In early stages of seed development, the onset of LEC1 expression occurs in the endosperm, followed by mobilization of the LEC1 protein to the embryo, likely via the plasmodesmata (PD) in the suspensor cells. After entering the embryo, the endosperm-synthesized LEC1 overcomes the repressive chromatin constraints to trigger the de-repression of seed maturation-related transcription factor genes including LEC2, ABI3, FUS3, and LEC1 itself (L.A.F.L.). Subsequently, the L.A.F.L. transcription factors activate the expression of seed storage reserve synthesis genes, enabling normal seed maturation. In the lec 1 mutant seeds, the L.A.F.L. network remains repressed due to lack of $L E C 1$ expression in both the endosperm and the embryo, thus leading to dramatically reduced accumulation of seed storage reserves and consequently the formation of desiccation-intolerant purple seeds. Notes: endosperm (Endo), embryo (Em), and suspensor (Sp) are colored with light amber, light green, and light blue, respectively; amber circles represent endosperm nuclei; dark green circles indicate embryo nuclei; orange and gray circles represent wild type and mutant LEC1 proteins, respectively; black lines represent genomic DNA and the orange/black rectangular bars indicate the wild type and mutant $L E C 1$ genes, respectively; purple circles represent histones; red stars represent repressive histone modifications, such as H3K27me3; and bright green squares represent seed storage reserves (SSPs: seed storage proteins).

that embryo development, in particular the maturation program, would not initiate until it is informed to do so, i.e., after successful fertilization, otherwise remains securely repressed during the rest of the plant's life cycle.

In conclusion, we demonstrate the critical importance of endosperm-synthesized LEC1 in enabling normal seed development including maturation in Arabidopsis. These findings establish LEC1 as a molecular signal in the communication between the embryo and the endosperm during seed development, and thus provide novel insights into the mechanisms by which the endosperm nourishes and regulates embryo development.

\section{Methods}

Plant materials and growth conditions. Arabidopsis thaliana ecotypes Columbia0 (Col-0) and Landsberg (Ler-0) were used as wild types. Arabidopsis mutant lines lec1-1 (SALK_131219) ${ }^{27}$, lec1-2 (CS870475), lec1-3 (CS5739) ${ }^{31}, c d k a ; 1$ (SALK_ 106809.34.90.X) ${ }^{16,17}$, and fis2-6 (CS6998) were obtained from the Arabidopsis Biological Resource Center (ABRC). Haploid Inducer SeedGFP-HI line was a gift from UC Davis ${ }^{18,19}$. Transgenic plants of pLEC1::LEC1-GFP lec1 (PLL), pPHE:: LEC1-GFP lec1 (PPL), pPHE::GFP lec1 (PPg), pPHE::ABI3-GFP lec1 (PPA), pZOU:: LEC1-GFP lec1 (PZL), pZOU::GFP lec1 (PZg), pZOU::ABI3-GFP lec1 (PZA), pPHE:: LEC1-GFP-GFP-GFP lec1 (PPL-GFP ${ }_{3}$ ), and pAtML1::LEC1-GFP lec1 (PML) were generated in this work. Note: lec1 can be either lec1-1 or lec1-3, as specified in the main text. Double mutant lines lec1-1 $c d k a ; 1$ and lec1-1 fis2-6 were produced by genetic crosses. The wild type and mutant seeds were germinated either in soil or on half-strength MS medium and grown in a growth room with humidity of $65 \%$ under a $16 \mathrm{~h}$ light $/ 8 \mathrm{~h}$ dark cycle at $22^{\circ} \mathrm{C}$. All genotypes were determined by PCR, by resistance to hygromycin, or by phenotype. Primers used for genotyping are listed in Supplementary Table 1.

Plasmid construction. To generate the pLEC1::LEC1-GFP construct, the LEC1 genomic region from $2000 \mathrm{bp}$ upstream of the ATG to the end of ORF without stop codon was amplified from Col-0 genomic DNA with primers F-pro-LEC1/R-LEC1, transferred into $\mathrm{pMDC} 107^{32}$. To generate the $p P H E:: L E C 1-G F P$ construct, the $L E C 1$ genomic region from ATG to the end of ORF without stop codon was amplified from Col-0 genomic DNA with primers F-LEC1/R-LEC, ligated into pMDC107 to get the LEC1-GFP construct. Then the PHE promoter region (2000 bp upstream of the ATG) was amplified from Col-0 DNA with primers F-pro-PHE/ $R$-pro-PHE, transferred to the LEC1-GFP construct. To build the pPHE::LEC1GFP-GFP-GFP construct, GFP-GFP was amplified with primers Pme1-Mlu1-GFP F/Asc1-GFP-R, transferred to pMDC107 to get the GFP-GFP-GFP construct. Then pPHE1::LEC1 was cloned with primers F-pro-PHE/Mlu1-LEC1-R, then transferred to the GFP-GFP-GFP construct. Similarly, to get the construct $p P H E:: A B I 3-G F P$, the $A B I 3$ genomic region from ATG to the end of ORF without stop codon was amplified from Col-0 genomic DNA with primers $F-A B I 3 / R-A B I 3$, then ligated into pMDC107 to get the $A B I 3-G F P$ construct before introducing the $P H E$ promoter amplified previously. To get the $p P H E:: G F P$ construct, the PHE promoter region (2000 bp upstream of the ATG) was amplified from Col-0 DNA with primers $F$ pro-PHE/R-pro-PHE-1, ligated into pMDC107. To generate the pZOU::LEC1-GFP construct, the $Z O U$ promoter region (2000 bp upstream of the ATG) was amplified from Col-0 DNA with primers F-pro-ZOU/R-pro-ZOU, then ligated to the LEC1GFP construct. To get the construct $p Z O U:: A B I 3-G F P$, the $Z O U$ promoter amplified previously was cloned into ABI3-GFP. To get the $p Z O U:: G F P$ construct, the $Z O U$ promoter region (2000 bp upstream of the ATG) was amplified from Col0 DNA with primers F-pro-ZOU/R-pro-ZOU-1, ligated into pMDC107. To generate the $p A t M L 1:: L E C 1-G F P$ construct, the AtML1 promoter region ${ }^{25}$ was amplified from Col-0 DNA with primers $p A t M L 1-F / p A t M L 1-R$, then ligated to the LEC1GFP construct. At least five independent transgenic lines for each construct 
transformation were obtained and examined. Primer information is listed in Supplementary Table 1. Plant transformation was performed via floral dipping ${ }^{33}$.

Seed rescue. Immature siliques from $l e c 1$ heterozygous parental plants were collected and surface sterilized with $70 \%$ ethanol, dissected under a dissecting microscope. Homozygous mutant seeds (purple color, with defective embryos) were transferred to half-strength MS agar plates with $1 \%$ sucrose for germination. After germination, seedlings were then transferred to soil for further experiments. lec1 homozygosity was normally confirmed by PCR-based genotyping.

\section{Genetic crosses for the fis2-cdka;1 bypassing assay and haploid seed pro-} duction. To obtain the lec1-1-1- fis $2-6^{+/-}$line, the lec1-1 homozygous mutant and fis $2-6$ heterozygous mutant plants were used for cross. The lec $1-1^{-1-}$ flowers at stage 12 were emasculated and shortly after that the pistils were hand-pollinated with pollen grains from the fis $2-6^{+/-}$plants. F1 seeds from the cross were collected and planted to produce F2 seeds. The lec1-1 $1^{-1-}$ fis $2-6^{+1-}$ plants were selected based on the combination of phenotypes of $l e c 1-1^{-1-}$ (purple color, not fully developed embryos) and fis $2-6^{+/-}$ (aborted seeds at heart stage) from the F2 self-crossed siliques. Similarly, the lec1-1 $1^{-1-}$ $c d k a ; 1^{+l-}$ line was obtained by crossing the lec1-1 $1^{-1-}$ line and the $c d k a ; 1^{+/-}$line. The $c d k a ; 1^{+1-}$ flowers at stage 12 were emasculated and the pistils were hand-pollinated with pollen grains from the lec1-1 $1^{-I-}$ plants. F1 seeds from the crossed siliques were used to produce F2 seeds. The lec $1-1^{-l-} c d k a ; 1^{+-}$plants were confirmed by the combination of phenotypes of lec1-1-l- (purple color, not fully developed embryo) and $c d k a ; 1^{+/-}$(half amount of seeds aborted at heart stage in one silique) from the F2 self-crossed siliques. For FIS-CDKA bypassing assays, fis $2-6^{+/-}$and $l e c 1-1^{-1-}$ fis $2-6^{+/-}$lines were used as maternal parents while $c d k a ; 1^{+1-}$ and $l e c 1-1^{-1-} c d k a ; 1^{+1-}$ plants were used as pollen donor. For SeedGFP-HI experiments, the SeedGFP-HI flowers were emasculated at stage 12 and hand-pollinated with pollen grains from the lec1-1 ${ }^{-1-}$ plants. Seeds were collected from the F1 siliques for further experiments.

Microscopy. For examination of seed morphology, siliques were hand-dissected for differential interference contrast (DIC) imaging by using a stereomicroscope For examination of embryo development, seeds were mounted in the Hoyer's solution for DIC ${ }^{34}$. The GFP florescence of seeds and embryos produced from the HI crosses were examined under UV lights with a stereomicroscope. The stereomicroscope used for the experiments was a Nikon SM225 equipped with a DS-Ri2 camera (Nikon). Confocal images were taken using an Olympus Fluoview FV1200 laser scanning microscope with excitation wavelength of $488 \mathrm{~nm}$ for EGFP and 559 $\mathrm{nm}$ for propidium iodide (PI). Confocal images were analyzed using the imaging software: OLYMPUS FLUOVIEW Ver.4.2.

RT-qPCR analysis. For RNA isolation, linear embryos were dissected as described previously with some modifications ${ }^{35}$. More specifically, individual embryos were hand-dissected under a stereomicroscope (EMZ PLS-2 stand, MEIJI) from seeds immersed in $10 \%$ RNAlater (Thermo Fisher Scientific) with fine point tweezers, and transferred with a 2-20 $\mu \mathrm{l}$ RNAse-free pipette tip (VWR) to depression slides (VWR) containing $200 \mu \mathrm{l}$ of $10 \%$ RNAlater. After every 10 embryos dissected, the embryos were washed with $10 \%$ RNAlater three times, then transferred to $30 \mu \mathrm{l}$ of $100 \%$ RNAlater. RNA was isolated from each pool of 50 embryos per sample by adding $500 \mu \mathrm{l} \mathrm{TRIzol}$ (Thermo Fisher Scientific) followed by incubation at $60^{\circ} \mathrm{C}$ for $30 \mathrm{~min}$, and then purified according to the TRIzol reagent protocol for RNA isolation from small quantities of tissue (Life Technologies). For each sample, $100 \mathrm{ng}$ of RNA was used in reverse transcription reactions using a iScript Reverse Transcription Supermix for RT-qPCR kit (Bio-RAD). For each quantification-PCR (qPCR), SsoFast EvaGreen Supermix (Bio-RAD) with Gene-specific and CACS (endogenous control) ${ }^{36}$ primers were used to conduct $\mathrm{qPCR}$ reactions on a BioRAD C1000 TM Thermal Cycler with the CFX96 ${ }^{\mathrm{TM}}$ Real-time PCR System (BioRAD). qPCR data was analyzed using Bio-Rad CFX Manager program. Primer information is listed in Supplementary Table 1.

Storage protein analysis. Mature seeds were ground in the extraction buffer (100 $\mathrm{mM}$ Tris- $\mathrm{HCl} \mathrm{pH} 8.0,1 \%$ SDS, $10 \%$ glycerol, and $2 \% \beta$-mercaptoethanol). The extracts were boiled for $3 \mathrm{~min}$, followed by centrifugation at $20,000 \mathrm{~g}$ for $5 \mathrm{~min}$. The supernatant of each sample was transferred to a new tube. Protein samples were mixed with $5 \times$ loading buffer, denatured by adding $18.5 \mathrm{mM}$ dithiothreitol, before loading onto $15 \%$ SDS-PAGE gels. After separation by electrophoresis using a Biochrom Novaspec Plus Visible Spectrophotometer (Bio-RAD), the protein gels were stained with Coomassie Brilliant Blue R250 for $30 \mathrm{~min}$, followed by destaining for $1 \mathrm{~h}$ with de-staining solution (10\% glacial acid, $40 \%$ methanol) before imaging with a GS-900 Calibrated Densitometer scanner.

Statistical analysis. R v.3.6.3 (http://www.r-project.org/) was used for the statistical analyses. Bartlett tests were performed to verify the equality of the variance across the samples. One-way ANNOVA analyses and post-hoc Tukey tests were conducted to determine the significant difference. Bar graphs were generated by using Microsoft Excel 2016.
Reporting summary. Further information on research design is available in the Nature Research Reporting Summary linked to this article.

\section{Data availability}

All lines used in the study will be provided upon signature of appropriate material transfer agreement. All data are available in the main text or the supplementary materials. Source data are provided with this paper.

Received: 26 August 2020; Accepted: 3 June 2021; Published online: 25 June 2021

\section{References}

1. Goldberg, R. B., De Paiva, G. \& Yadegari, R. Plant embryogenesis: zygote to seed. Science 266, 605-614 (1994).

2. Lafon-Placette, C. \& Köhler, C. Embryo and endosperm, partners in seed development. Curr. Opin. Plant Biol. 17, 64-69 (2014).

3. Li, J. \& Berger, F. Endosperm: food for humankind and fodder for scientific discoveries. New Phytol. 195, 290-305 (2012).

4. Doll, N. M. et al. A two-way molecular dialogue between embryo and endosperm is required for seed development. Science 367, 431-435 (2020).

5. Jo, L., Pelletier, J. M. \& Harada, J. J. Central role of the LEAFY COTYLEDON1 transcription factor in seed development. J. Integr. Plant Biol. 61, 564-580 (2019).

6. $\mathrm{Wu}$, J. et al. Spatiotemporal restriction of FUSCA3 expression by class I BPCs promotes ovule development and coordinates embryo and endosperm growth. Plant Cell 32, 1886-1904 (2020).

7. Luerßen, H., Kirik, V., Herrmann, P. \& Miséra, S. FUSCA3 encodes a protein with a conserved VP1/AB13-like B3 domain which is of functional importance for the regulation of seed maturation in Arabidopsis thaliana. Plant J. 15, 755-764 (1998).

8. Stone, S. L. et al. LEAFY COTYLEDON2 encodes a B3 domain transcription factor that induces embryo development. Proc. Natl Acad. Sci. USA 98, 11806-11811 (2001)

9. Giraudat, J. et al. Isolation of the Arabidopsis ABI3 gene by positional cloning. Plant Cell 4, 1251-1261 (1992).

10. Pelletier, J. M. et al. LEC1 sequentially regulates the transcription of genes involved in diverse developmental processes during seed development. Proc. Natl. Acad. Sci. USA https://doi.org/10.1073/pnas.1707957114 (2017).

11. West, M. A. L. et al. LEAFY COTYLEDON1 is an essential regulator of late embryogenesis and cotyledon identity in Arabidopsis. Plant Cell 6, 1731-1745 (1994).

12. Meinke, D. W., Franzmann, L. H., Nickle, T. C. \& Yeung, E. C. Leafy cotyledon mutants of Arabidopsis. Plant Cell 6, 1049-1064 (1994).

13. Belmonte, M. F. et al. Comprehensive developmental profiles of gene activity in regions and subregions of the Arabidopsis seed. Proc. Natl Acad. Sci. USA 110, E435-E444 (2013).

14. Huang, Y. et al. Probing the endosperm gene expression landscape in Brassica napus. BMC Genomics 10, 256 (2009).

15. Lotan, T. et al. Arabidopsis LEAFY COTYLEDON1 is sufficient to induce embryo development in vegetative cells. Cell 93, 1195-1205 (1998).

16. Nowack, M. K. et al. Bypassing genomic imprinting allows seed development. Nature 447, 312-316 (2007).

17. Nowack, M. K. et al. A positive signal from the fertilization of the egg cell sets off endosperm proliferation in angiosperm embryogenesis. Nat. Genet. 38, 63-67 (2006)

18. Ravi, M. et al. A haploid genetics toolbox for Arabidopsis thaliana. Nat. Commun. 5, 1-8 (2014).

19. Ravi, M. \& Chan, S. W. L. Haploid plants produced by centromere-mediated genome elimination. Nature 464, 615-619 (2010)

20. Moreno-Romero, J., Santos-González, J., Hennig, L. \& Köhler, C. Applying the INTACT method to purify endosperm nuclei and to generate parental-specific epigenome profiles. Nat. Protoc. 12, 238-254 (2017).

21. Köhler, C., Page, D. R., Gagliardini, V. \& Grossniklaus, U. The Arabidopsis thaliana MEDEA polycomb group protein controls expression of PHERES1 by parental imprinting. Nat. Genet. 37, 28-30 (2005).

22. Yang, S. et al. The endosperm-specific ZHOUPI gene of Arabidopsis thaliana regulates endosperm breakdown and embryonic epidermal development. Development 135, 3501-3509 (2008).

23. Clark, N. M. et al. Tracking transcription factor mobility and interaction in arabidopsis roots with fluorescence correlation spectroscopy. Elife 5, 1-25 (2016).

24. Kim, I., Kobayashi, K., Cho, E. \& Zambryski, P. C. Subdomains for transport via plasmodesmata corresponding to the apical-basal axis are established during Arabidopsis embryogenesis. Proc. Natl Acad. Sci. USA 102, 11945-11950 (2005) 
25. Tsuchiya, Y., Nambara, E., Naito, S. \& McCourt, P. The FUS3 transcription factor functions through the epidermal regulator TTG1 during embryogenesis in Arabidopsis. Plant J. 37, 73-81 (2004).

26. To, A. et al. A network of local and redundant gene regulation governs Arabidopsis seed maturation. Plant Cell 18, 1642-1651 (2006).

27. Tao, Z. et al. Embryonic epigenetic reprogramming by a pioneer transcsription factor in plants. Nature 551, 124-128 (2017).

28. Li, C. et al. The Arabidopsis SWI2/SNF2 chromatin remodeler BRAHMA regulates polycomb function during vegetative development and directly activates the flowering repressor gene SVP. PLoS Genet. 11, 1-25 (2015).

29. Tang, X. et al. The Arabidopsis BRAHMA chromatin-remodeling ATPase is involved in repression of seed maturation genes in leaves. Plant Physiol. 147, 1143-1157 (2008).

30. Shu, J. et al. Genome-wide occupancy of histone H3K27 methyltransferases CURLY LEAF and SWINGER in Arabidopsis seedlings. Plant Direct 3, 1-14 (2019).

31. $\mathrm{Hu}, \mathrm{Y}$. et al. Gibberellins play an essential role in late embryogenesis of Arabidopsis. Nat. Plants 4, 289-298 (2018).

32. Curtis, M. D. \& Grossniklaus, U. A gateway cloning vector set for high-throughput functional analysis of genes in planta. Plant Physiol. 133, 462-469 (2003).

33. Zhang, X., Henriques, R., Lin, S., Niu, Q. \& Chua, N. Agrobacterium-mediated transformation of Arabidopsis thaliana using the floral dip method. Nat. Protoc. 1, 1-6 (2006)

34. Berleth, T. \& Jurgens, G. The role of the monopteros gene in organising the basal body region of the Arabidopsis embryo. Development 118, 575-587 (1993).

35. Nodine, M. D. \& Bartel, D. P. MicroRNAs prevent precocious gene expression and enable pattern formation during plant embryogenesis. Gene Dev. 24, 2678-2692 (2010).

36. MacGregor, D. R. et al. ICE1 and ZOU determine the depth of primary seed dormancy in Arabidopsis independently of their role in endosperm development. Plant J. 98, 277-290 (2019).

\section{Acknowledgements}

We thank the Arabidopsis Biological Resource Center for providing the mutant seeds used in this study, and Dr. Maruthachalam Ravi and Dr. Mohan Marimuthu (University of California, Davis) for kindly providing us the SeedGFP-HI seeds. This work was supported by grants from the Natural Science and Engineering Research Council of Canada (RGPIN/04625-2017 to Y.C.), Agriculture and Agri-Food Canada (to Y.C.), and the Sustainable Food System program of Aquatic and Crop Resource Development Research Centre, National Research Council of Canada (to J.Z.).

\section{Author contributions}

J.Z. and Y.C. conceived the project; C.C., Y.C., J. Song, and J.Z. designed the experiments; J. Song conducted most of the experiments; X.X. generated the PPL-GFP ${ }_{3}$ and PML lines, propagated transgenic lines, and conducted genotyping. V.N. performed the storage protein extraction experiment; J. Shu and R.T. contributed to the genetic work and molecular analyses; S.B., S.K., and F.M. contributed to data analysis and supervision; J. Song, J.Z., and Y.C. wrote the manuscript.

\section{Competing interests}

The authors declare no competing interests.

\section{Additional information}

Supplementary information The online version contains supplementary material available at https://doi.org/10.1038/s41467-021-24234-1.

Correspondence and requests for materials should be addressed to J.S., J.Z. or Y.C.

Peer review information Nature Communications thanks the anonymous reviewers for their contribution to the peer review of this work. Peer review reports are available.

Reprints and permission information is available at http://www.nature.com/reprints Publisher's note Springer Nature remains neutral with regard to jurisdictional claims in published maps and institutional affiliations.

(c) (i) Open Access This article is licensed under a Creative Commons Attribution 4.0 International License, which permits use, sharing, adaptation, distribution and reproduction in any medium or format, as long as you give appropriate credit to the original author(s) and the source, provide a link to the Creative Commons license, and indicate if changes were made. The images or other third party material in this article are included in the article's Creative Commons license, unless indicated otherwise in a credit line to the material. If material is not included in the article's Creative Commons license and your intended use is not permitted by statutory regulation or exceeds the permitted use, you will need to obtain permission directly from the copyright holder. To view a copy of this license, visit http://creativecommons.org/ licenses/by/4.0/.

(c) Crown 2021 